\title{
Study on OSPF Routing Protocol of Computer Network Based on Packet Tracer
}

\author{
Henghua SHI ${ }^{1, a}$, Yujie WANG ${ }^{1, b^{*}}$, Renlong ZHANG ${ }^{1, \mathrm{c}^{*}}$ \\ ${ }^{1}$ School of Computer and Information Engineering, Beijing University of Agriculture, China \\ ahenghuashi@163.com, ${ }^{b}$ wyj1119@bac.edu.cn, ${ }^{b}$ zrl@bac.edu.cn ${ }^{\text {bemail }}$ \\ * please mark the corresponding author with an asterisk
}

Keywords: Routing Protocol; OSPF; Packet Tracer

\begin{abstract}
The routing protocol is the most important network technology such as RIP, OSPF, etc. OSPF is a Link State Routing Protocol and widely used in more and more large networks. In this paper, we study Packet Tracer as one education simulation technology, and configure OSPF with single region in a simulation network topology. The simulate results show that we can easily configure OSPF in simulation network topology for learning and teaching, and can achieve the same efficient as in actual network equipments.
\end{abstract}

\section{Introduction}

The connection is the most important function of computer network, and the routing protocol is the key of the network connection. Routing protocol specify how routers communicate with each other, disseminating information that enables them to select routes between any two nodes on a computer network. Routing protocol shares this information first among immediate neighbors, and then throughout the network. Then, routers gain knowledge of the topology of the network by this way. Routing algorithms determine the specific choice of route. Each router has a priori knowledge only of networks attached to it directly.

There are many types of routing protocols, and those routing protocols are divided with three major classes: Interior Gateway Protocols [1] type 1 and type 2, Exterior gateway protocols. Interior Gateway Protocols type 1 is link-state routing protocols, such as OSPF [2] and IS-IS [3]. Interior gateway protocols type 2 is distance-vector routing protocols, such as Routing Information Protocol [4], RIPv2, IGRP [5]. Exterior gateway protocols are routing protocols used on the Internet for exchanging routing information between Autonomous Systems [6], such as BGP [7].

Many colleges and universities have the computer network class and teach the network connection technology such as routing protocol. For OSPF widely used in more and more large networks, OSPF is the main knowledge in routing protocol teaching. In this paper, we simulate a computer network topology and configure OSPF based on Packet Tracer for learning and teaching routing protocol. We analyze the simulation result and compare the advantage of education simulation technology such as with actual network equipments.

\section{Packet Tracer}

Packet Tracer [8] is one of the most popular and the easiest simulation tool for Cisco Systems, Inc. It is a powerful network simulation program that allows students to experiment with network behavior, and provides simulation, visualization, and authoring, assessment, and collaboration capabilities and facilitates the teaching and learning of complex technology concepts.

Packet Tracer is as a education simulation technology and has been widely used in many research including teaching method research [9][10]. Packet Tracer supplements physical equipment in the classroom by allowing students to create a network with an almost unlimited number of devices, encouraging practice, 
discovery, and troubleshooting. The simulation-based learning environment helps students develop 21st century skills such as decision making, creative and critical thinking, and problem solving.

\section{OSPF Routing Protocol}

OSPF (Open Shortest Path First) is a routing protocol for IP networks. It uses a link state routing algorithm and falls into the group of interior routing protocols, operating within a single autonomous system (AS). OSPF is perhaps the most widely used Interior Gateway Protocols in large enterprise networks.

OSPF does not use a TCP/IP transport protocol, such as UDP or TCP, but encapsulates its data in IP datagrams with protocol number 89 . This is in contrast to other routing protocols, such as the RIP and the BGP. OSPF implements its own error detection and correction functions.

The OSPF routing policies for constructing a route table are governed by link cost factors (external metrics) associated with each routing interface. Cost factors may be the distance of a router (round-trip time), data throughput of a link, or link availability and reliability, expressed as simple unit less numbers. This provides a dynamic process of traffic load balancing between routes of equal cost.OSPF detects changes in the topology, such as link failures, and converges on a new loop-free routing structure within seconds. It computes the shortest path tree for each route using a method based on Dijkstra's algorithm, a shortest path first algorithm.

\section{Simulation Network Experiment}

In Fig. 1., we simulate a network topology includes three routers and six PCs as six LANs with Packet Tracer. Then, we configure the simulation topology as Table 1 .

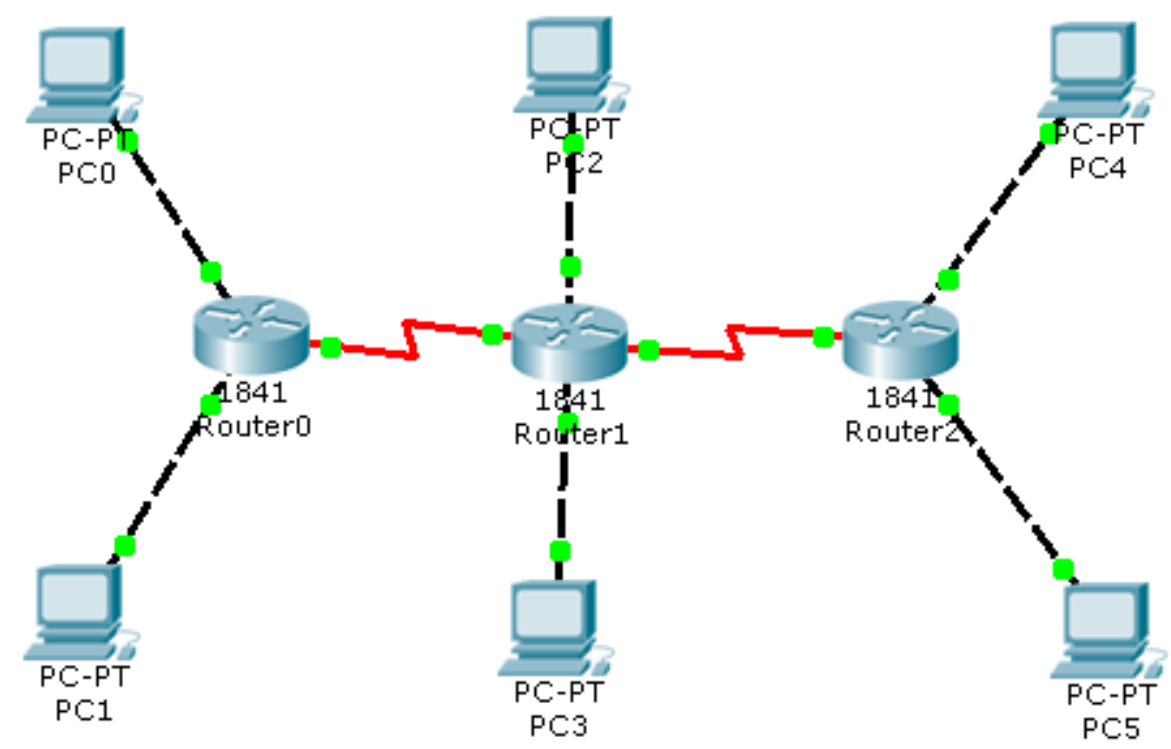

Fig. 1 The simulation topology

We configure IP address for PC0, PC1, PC2, PC3, PC4, and PC5 and configure interfaces such as Loopback, $\mathrm{Fa} 0 / 0, \mathrm{Fa} 0 / 1$, and S0/0/0 of Router0 with Table1. The command on Router0 is as following:

Router0(config)\#interface loopback 0

Router0(config-if)\#ip address 1.1.1.1 255.255.255.255

Router0(config-if)\#no shutdown

Router0(config-if)\#interface fastEthernet 0/0

Router0(config-if)\#ip address 192.168.1.254 255.255.255.0

Router0(config-if)\#no shutdown 
Router0(config-if)\#interface fastEthernet 0/1

Router0(config-if)\#ip address 192.168.2.254 255.255.255.0

Router0(config-if)\#no shutdown

Router0(config-if)\#interface serial 0/0/0

Router0(config-if)\#ip address 192.168.10.2 255.255.255.0

Router0(config-if)\#no shutdown

Router0(config-if)\#router ospf 111

Router0(config-router)\#network 192.168.1.0 0.0.0.255 area 0

Router0(config-router)\#network 192.168.2.0 0.0.0.255 area 0

Router0(config-router)\#network 192.168.10.0 0.0.0.255 area 0

Router0(config-router)\#end

Table 1 The IP address of network topology

\begin{tabular}{lllll}
\hline Device & Interface & IP address & Subnet Mask & Default Gatway \\
\hline PC0 & NIC & 192.168 .1 .1 & 255.255 .255 .0 & 192.168 .1 .254 \\
PC1 & NIC & 192.168 .2 .1 & 255.255 .255 .0 & 192.168 .2 .254 \\
PC2 & NIC & 192.168 .3 .1 & 255.255 .255 .0 & 192.168 .3 .254 \\
PC3 & NIC & 192.168 .4 .1 & 255.255 .255 .0 & 192.168 .4 .254 \\
PC4 & NIC & 192.168 .5 .1 & 255.255 .255 .0 & 192.168 .5 .254 \\
PC5 & NIC & 192.168 .6 .1 & 255.255 .255 .0 & 192.168 .6 .254 \\
Router0 & Loopback & 1.1 .1 .1 & 255.255 .255 .255 & N/A \\
& Fa0/0 & 192.168 .1 .254 & 255.255 .255 .0 & N/A \\
& Fa0/1 & 192.168 .2 .254 & 255.255 .255 .0 & N/A \\
& S0/0/0 & 192.168 .10 .2 & 255.255 .255 .0 & N/A \\
Router1 & Loopback & 2.2 .2 .2 & 255.255 .255 .255 & N/A \\
& Fa0/0 & 192.168 .3 .254 & 255.255 .255 .0 & N/A \\
& Fa0/1 & 192.168 .4 .254 & 255.255 .255 .0 & N/A \\
& S0/0/0 & 192.168 .10 .1 & 255.255 .255 .0 & N/A \\
& S0/0/1 & 192.168 .20 .1 & 255.255 .255 .0 & N/A \\
Router2 & Loopback & 3.3 .3 .3 & 255.255 .255 .255 & N/A \\
& Fa0/0 & 192.168 .5 .254 & 255.255 .255 .0 & N/A \\
& Fa0/1 & 192.168 .6 .254 & 255.255 .255 .0 & N/A \\
& S0/0/1 & 192.168 .20 .2 & 255.255 .255 .0 & N/A
\end{tabular}

In the same way as the configuration of Router0, we also configure interfaces of Router1 and Router2 with Table 1. After the above configuration, all PCs can communicate with each other. For example, PC0 can connect to PC5 with ping command. The results show as Fig. 2 in Smulation Mode of Packet Tracer. 


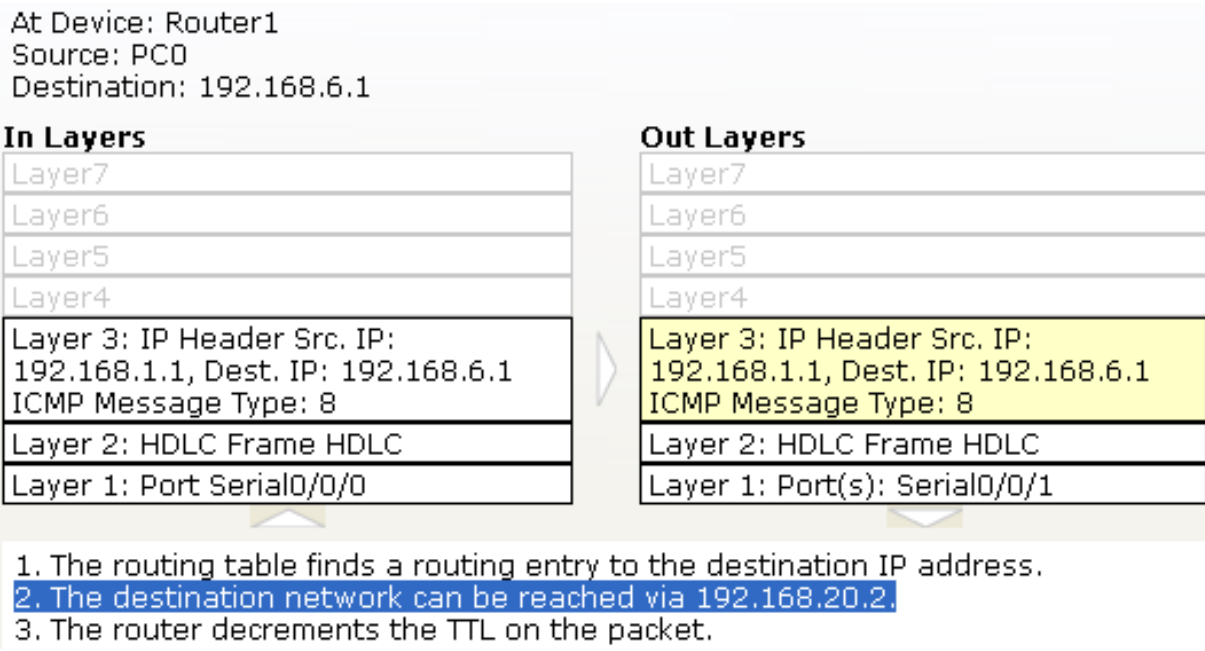

Fig. 2 Communicate between PC0 and PC1

The dark mark line is means that ICMP message from PC0 to PC5 is translate via 192.168.20.2 by OSPF routing protocol. We can check the corresponding OSPF routing entry on Router1 with show ip route command as following:

Router1\# show ip route

O $192.168 .1 .0 / 24[110 / 65]$ via 192.168.10.2, 00:02:10, Serial0/0/0

O 192.168.2.0/24 [110/65] via 192.168.10.2, 00:02:10, Serial0/0/0

C $192.168 .3 .0 / 24$ is directly connected, FastEthernet $0 / 0$

C 192.168.4.0/24 is directly connected, FastEthernet $0 / 1$

O $192.168 .5 .0 / 24[110 / 65]$ via 192.168.20.2, 00:00:33, Serial0/0/1

O 192.168.6.0/24 [110/65] via 192.168.20.2, 00:00:33, Serial0/0/1

C $192.168 .10 .0 / 24$ is directly connected, Serial0/0/0

C $192.168 .20 .0 / 24$ is directly connected, Serial0/0/1

\section{Summary}

In colleges and universities teaching lesson, the routing protocol is one of the most important knowledge for the network connection. We select OSPF routing protocol as the representative of Interior Gateway Protocols with link-state routing protocols, and configure OSPF based on Packet Tracer as an education simulation technology. The simulation results show we can achieve configuration task with Packet Tracer replacing the actual network equipments, and can study OSPF routing protocol more easily and efficiently. Comparing with the actual network equipments, we can see the education simulation technology such as Packet Tracer can approve more easily and efficiently method for learning and teaching.

\section{Acknowledgement}

Corresponding author is WANG Yujie. The authors would like to acknowledge the supports provided by 2015 Research Fund for Academic Degree \& Graduate Education of Beijing University of Agriculture (2015YJS035).

\section{References}

[1] B. Y. Bie. IGP Routing Design and Analysis of Large-scale IP Network Engineering. The Second Annual Conference, Institute of Communications in 2006 Proceedings, Wuhan, China, 2006: 259-262

[2] J. Moy, The Internet Society. OSPFv2. Retrieved 2007-09-28. 
[3] H. Gredler, W. Goraiski, The complete IS-IS routing protocol. Springer. 2005.

[4] C. Hedrick, Routing Information Protocol .IETF RFC 1058. 1988.

[5] Cisco Systems. An Introduction to IGRP. Document ID: 26825, 1991-08-22.

[6] J. Hawkinson, Network Working Group. IETF RFC 1930. 1996.

[7] Y. Rekhter, A Border Gateway Protocol 4 (BGP-4). RFC 4271, 2006

[8] Cisco Packet Tracer, https://www.netacad.com/web/about-us/cisco-packet-tracer.

[9] D. C. Frezzo, J. T. Behrens, R. J. Mislevy, P. West, K. E. DiCerbo, Psychometric and evidentiary approaches to simulation assessment in Packet Tracer software. Proceedings of the 5th International Conference on Networking and Services, ICNS 2009. pp,555-560.

[10] D. Petcu, B. Iancu, A. Peculea, V. Dadarlat, E. Cebuc, Integrating Cisco Packet Tracer with Moodle platform: Support for teaching and automatic evaluation. 12th RoEduNet International Conference: Networking in Education and Research, RoEduNet 2013. 